\title{
Collaborative Voltage Unbalance Elimination in Grid-Connected AC Microgrids with Grid- Feeding Inverters
}

\author{
Ángel Borrell, Manel Velasco, Jaume Miret, member IEEE, Antonio Camacho, Pau Martí and Miguel Castilla
}

\begin{abstract}
The control flexibility of the power inverters that interface distributed generation can be exploited to incorporate power quality enhancement features in addition to the usual power management control. This paper presents a novel control scheme that achieves voltage balance at any node of an AC microgrid regardless of the impedance value of the power network. The collaborative strategy is devoted to set a specific sharing of the balancing currents, thus avoiding the adverse effects of the unbalance compensation by a single inverter. The control scheme uses a complex form of a proportional resonant controller and a communication link. The paper presents the stability and sensitivity analysis and the design guidelines for the control parameters. The performance of the control proposal has been tested successfully in a laboratory microgrid setup. Selected experimental results are presented.
\end{abstract}

Index Terms-Distributed generation, grid-feeding converter, voltage unbalance, power quality, microgrids.

\section{INTRODUCTION}

$\mathrm{V}$ OLTAGE unbalance occurs when voltages of a threephase system are not identical in magnitude and/or the phase differences between them are not exactly $120^{\circ}$ [1]. The causes of voltage unbalance can be faults in the generation side, asymmetrical transformer winding impedances, mismatched transmission lines, the connection of single-phase photovoltaic inverters, plug-in electric vehicles and uneven distribution of single-phase loads [2], [3]. Unbalanced voltages can result in adverse effects on the power system, such as more losses and less stability. The effect of voltage unbalance can also be severe on equipment, such as induction motors, power electronic converters, and adjustable speed drives [4]-[8]. The voltage unbalance factor (VUF), i.e. the percentage ratio of the negative-sequence voltage component to the positive-sequence voltage component, is widely used to characterize the unbalance [9]. The standard EN50160 specifies a VUF < 2\% for medium and low voltage systems [10]. Usually, the voltage unbalance is mitigated with dedicated equipment such as series/shunt active power filters, dynamic voltage restorers, or static synchronous compensators [11]-[14].

Nowadays microgrids are dominated by electronically interfaced distributed generation units operating in gridfeeding or grid-forming mode. The flexibility of the power electronics interface can be exploited to incorporate power quality enhancement features in addition to the usual power management control [15], [16]. This potential idea relies on two facts. First, most interfacing converters are not operating at full rating all the time due to the intermittent nature of renewable energy sources. Second, the deployment of communication links in power systems facilitates the implementation of collaborative control strategies to address power quality issues without oversizing the power electronics interface.

There are some control approaches for voltage unbalance mitigation implemented in the distributed generation power interface [17]-[21]. All of them have in common that the control scheme works with local variables (output voltage and current). Therefore, the voltage unbalance mitigation is limited to the node where the inverter is connected. In [17], a cooperative approach with the droop control method based on the reactive power produced by the negative-sequence current is proposed. However, the power line impedance affects the accuracy of the negative-sequence reactive power sharing of the droop control. In the proposal presented in [18], the compensation reference is obtained from the negativesequence reactive power and a control gain, which is adjusted for sharing the compensation effort amongst distributed generators. Nevertheless, with this control approach, a tradeoff exists between the compensation level (not elimination) and stability. The work in [19] reduces the negative sequence voltage by injecting negative-sequence current in-phase with the grid negative-sequence current. However, the performance is affected by the grid impedance value. The control scheme presented in [20] relies on a virtual bus model and an adaptive integral compensator. The generated negative-sequence current reference practically achieves voltage unbalance elimination but only at the converter output. The authors in [21] present a control solution that eliminates the voltage unbalance produced by the grid at the inverter output regardless of the grid impedance value.

This paper presents a novel control scheme to address the limitations of the published works. The three objectives of this work are: an accurate voltage unbalance elimination at any point of the AC power network, a collaborative strategy to share the necessary negative-sequence current amongst the participating units, and the achievement of the two previous objectives regardless of the grid impedance value. The control scheme eliminates the voltage unbalance introduced by the generation side, at any node of the AC power network, usually where sensitive loads are connected to, irrespective of the grid impedance value. This is the main contribution of this paper. This feature gives flexibility to the power network operator to meet customizable power quality requirements regardless of the location and the grid impedance value. Besides, the collaborative strategy is devoted to set a specific sharing of the balancing currents, thus avoiding the adverse effects of the 
TABLE I

PERFormanCE COMPARISON OF CONTROL SCHEMES WITH VOLTAGE UnBalanCE REDUCTION

\begin{tabular}{|c|c|c|c|c|c|c|}
\hline \multirow[b]{2}{*}{ Concept } & \multicolumn{6}{|c|}{ Control Scheme } \\
\hline & {$[17]$} & {$[18]$} & [19] & {$[20]$} & {$[21]$} & proposal \\
\hline Voltage unbalance & reduction & reduction & reduction & elimination & elimination & elimination \\
\hline $\begin{array}{l}\text { Location of the voltage } \\
\text { unbalance }\end{array}$ & inverter output & inverter output & inverter output & inverter output & inverter output & any point \\
\hline $\begin{array}{l}\text { Negative-sequence current } \\
\text { sharing accuracy }\end{array}$ & low & low & & & & high \\
\hline $\begin{array}{l}\text { Grid impedance value } \\
\text { dependence }\end{array}$ & yes & yes & yes & yes & no & no \\
\hline
\end{tabular}

unbalance compensation by a single inverter. Additionally, this proposal is recommended to microgrids with a weak connection to the main grid and with a high number of converters. In this scenario, the current supplied by the converters does not overpass the maximum current. In other situations, such as those including few inverters and connection to the network with very low impedance, the solution to this problem requires the use of extra hardware (UPS, STATCOM), which increases the cost of the installation. Therefore, the proposal is a very useful low-cost solution in some applications.

To summarize, Table I compares the performance of the control schemes of the state-of-the-art overview [17]-[21] in front of the proposal of this paper. The comparison considers the level of achievement of the control action (reduction or elimination of the voltage unbalance), the location of the voltage unbalance (at the inverter output or any point in the power network), the negative-sequence current sharing accuracy for the solutions with collaborative approach (low or high) and the dependence of the controller on the grid impedance value (yes or no). As mentioned, the proposal in this paper eliminates the voltage unbalance, at any point in the power network, regardless of the grid impedance value and with accurate current sharing.

In terms of implementation, the control scheme uses a communication link and a complex form of a negativesequence proportional resonant controller [22] for achieving the three control objectives. The paper presents the stability analysis, the design guidelines for the control parameters, and the sensitivity analysis to determine the impact of variations of the plant on the controller performance. The control proposal has been tested successfully in a laboratory setup.

The rest of the paper is organized as follows. Section II formulates the problem. Section III presents the control proposal. Section IV focuses on the stability and sensitivity analysis and provides the control design guidelines. Section V shows selected experimental results. Finally, Section VI gives the conclusions.

\section{PROBLEM FORMULATION}

\section{A. Plant Model}

Fig. 1. shows a representation of a three-phase three-wire AC-microgrid. The cloud symbol represents the actual network topology to consider a general case to formulate the plant model. The three-phase network consists of a series of interconnected nodes. The three-wire line interconnecting two consecutive three-phase nodes presents matching line impedance. Balanced three-phase loads and power sources are distributed through the network. The $N$ power sources are interfaced to the network by $N$ three-phase inverters. Every inverter is modeled as a positive-negative sequence ideal current source. The $N$ inverters work in grid-feeding mode injecting positive-sequence current into the power network to meet their respective active-reactive power setpoint. The ACmicrogrid is connected to the main grid through an $R-L$ line at the point of common coupling (PCC). In the event the main grid presents a voltage unbalance, it will be transmitted through all the microgrid affecting the operation of a sensitive load located at the $k^{\text {th }}$ node. In this situation, the control proposal will make the $N$ inverters inject the necessary negative-sequence current to eliminate the voltage unbalance introduced by the main grid as an ancillary service.

The three-phase voltage vector at the $k^{\text {th }}$ node $\left(\vec{v}_{a, b, c}\right)_{k}$, where the sensitive load is located, can be compactly expressed in the Laplace domain as

$$
\left(\vec{v}_{a, b, c}\right)_{k}=\sum_{i=1}^{N} \boldsymbol{G}(s)_{i}\left(\vec{l}_{a, b, c}\right)_{i}+\boldsymbol{W}(s) \vec{v}_{g a, b, c}
$$

which describes the impact of both the injected inverter currents $\left(\vec{l}_{a, b, c}\right)_{i}(i=1 . . N)$ and the grid voltage $\vec{v}_{g a, b, c}$ on the voltage at the $k^{\text {th }}$ node. To make the notation clearer, the domain subscript (s) for the variables is omitted. Since the power lines and the loads are balanced, the $3 \times 3$ matrices $\boldsymbol{G}(s)_{i}(i=1 \ldots N)$ and $\boldsymbol{W}(s)$ are symmetric with diagonal and off-diagonal transfer functions $G_{r r}(s), G_{r t}(s)$, and $W_{r r}(s)$, $W_{r t}(s)$, respectively, $(r=1 . .3)$ and $(t=1 . .3)$. Their developed expressions will depend on the topology configuration of the power network and they can be obtained systematically by using modified nodal analysis (MNA) [23].

The voltages and currents $\vec{x}_{a, b, c}$ are transformed into the $\alpha \beta$ stationary reference frame using the $\alpha \beta 0$ transformation

$$
\vec{x}_{a, b, c}=\left(\begin{array}{l}
x_{a} \\
x_{b} \\
x_{c}
\end{array}\right)=\left(\begin{array}{ccc}
1 & 0 & 1 \\
-\frac{1}{2} & \frac{\sqrt{3}}{2} & 1 \\
-\frac{1}{2} & -\frac{\sqrt{3}}{2} & 1
\end{array}\right)\left(\begin{array}{l}
x_{\alpha} \\
x_{\beta} \\
x_{0}
\end{array}\right)=T \vec{x}_{\alpha, \beta, 0} .
$$




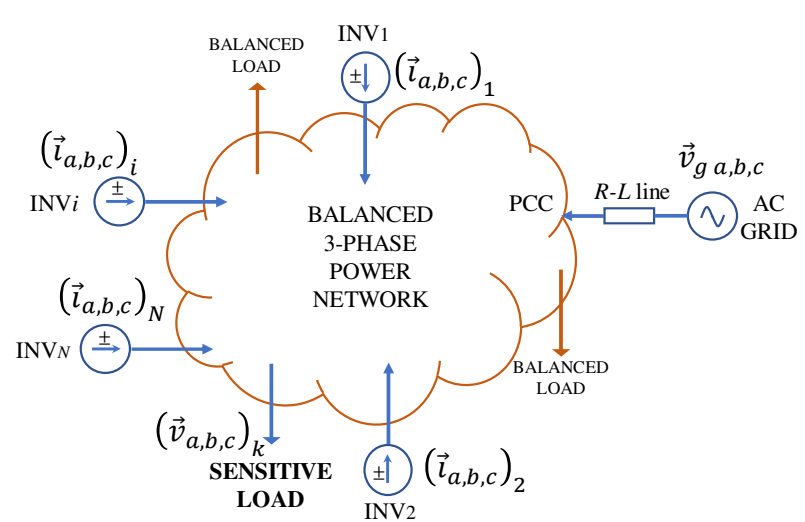

Fig. 1. Generalized three-phase AC-microgrid.

Applying (2) to the model described in (1) leads to the following plant expression

$$
\begin{aligned}
\left(\vec{v}_{\alpha, \beta, 0}\right)_{k} & =\sum_{i=1}^{N} T^{-1} \boldsymbol{G}(s)_{i} T\left(\vec{\imath}_{\alpha, \beta, 0}\right)_{i}+T^{-1} \boldsymbol{W}(s) T \vec{v}_{g \alpha, \beta, 0} \\
& =\sum_{i=1}^{N} \boldsymbol{G}_{\alpha, \beta, 0}(s)_{i}\left(\vec{\imath}_{\alpha, \beta, 0}\right)_{i}+\boldsymbol{W}_{\alpha, \beta, 0}(s) \vec{v}_{g \alpha, \beta, 0} .
\end{aligned}
$$

After applying the $\alpha \beta 0$ transformation, diagonal matrices $\boldsymbol{G}_{\alpha, \beta, 0}(s)_{i}(i=1 . . N)$ and $\boldsymbol{W}_{\alpha, \beta, 0}(s)$ are obtained. Their diagonal elements are $G_{\alpha \alpha}(s)=G_{\beta \beta}(s) \triangleq G(s)$ and $W_{\alpha \alpha}(s)=W_{\beta \beta}(s) \triangleq W(s)$, respectively.

In three-wire power lines the homopolar component is zero. Therefore, all the homopolar components of (3) can be removed. Expanding the variable vectors and matrices, the plant is equivalent to

$$
\begin{aligned}
\left(\begin{array}{l}
v_{\alpha} \\
v_{\beta}
\end{array}\right)_{k} & =\sum_{i=1}^{N}\left(\begin{array}{cc}
G(s) & 0 \\
0 & G(s)
\end{array}\right)_{i}\left(\begin{array}{l}
i_{\alpha} \\
i_{\beta}
\end{array}\right)_{i} \\
& +\left(\begin{array}{cc}
W(s) & 0 \\
0 & W(s)
\end{array}\right)\left(\begin{array}{l}
v_{g} \alpha \\
v_{g \beta} \beta
\end{array}\right) .
\end{aligned}
$$

At this point, the plant model is oriented to the control goal of eliminating the voltage unbalance introduced by the main grid at the $k^{\text {th }}$ node. Since the line and the load impedances are balanced, the $k^{\text {th }}$ node voltage given by (4) also holds for the positive and negative symmetrical components. Therefore, $G(s) \triangleq G^{-}(s)$ and $W(s) \triangleq W^{-}(s)$. By using the negativesequence component of (4), the plant model expressing the voltage unbalance at the $k^{\text {th }}$ node is given by

$$
\begin{aligned}
\left(\begin{array}{c}
v_{\alpha}^{-} \\
v_{\beta}^{-}
\end{array}\right)_{k} & =\sum_{i=1}^{N}\left(\begin{array}{cc}
G^{-}(s) & 0 \\
0 & G^{-}(s)
\end{array}\right)_{i}\left(\begin{array}{l}
i_{\alpha}^{-} \\
i_{\beta}^{-}
\end{array}\right)_{i} \\
& +\left(\begin{array}{cc}
W^{-}(s) & 0 \\
0 & W^{-}(s)
\end{array}\right)\left(\begin{array}{l}
v_{g}^{-} \alpha \\
v_{g}^{-} \beta
\end{array}\right)
\end{aligned}
$$

The $\alpha \beta$ negative components of voltages and currents are in quadrature and can be represented as complex variables

$$
\left(\begin{array}{l}
x_{\alpha}^{-} \\
x_{\beta}^{-}
\end{array}\right)=\left(x_{\alpha}^{-}+j x_{\beta}^{-}\right)=\left(x_{\alpha+j \beta}^{-}\right) \text {. }
$$

Hence, the plant model described by (5) can be written in complex form as

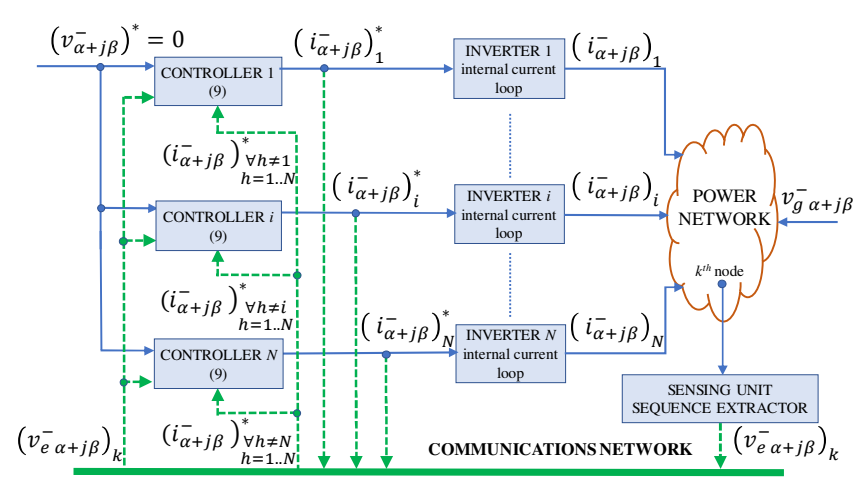

Fig. 2. Negative-sequence control scheme.

$$
\left(v_{\alpha+j \beta}^{-}\right)_{k}=\sum_{i=1}^{N} G^{-}(s)_{i}\left(i_{\alpha+j \beta}^{-}\right)_{i}+W^{-}(s) v_{g}^{-} \alpha+j \beta .
$$

\section{B. Problem Feasibility}

The goal is to make $\left(v_{\alpha+j \beta}^{-}\right)_{k}$ zero with the $N$ inverters injecting the necessary net negative-sequence current. With prior knowledge of $G^{-}(s)_{i}(i=1 . . N)$ and $W^{-}(s)$ and using the open-loop plant model expressed by (7), the problem of eliminating negative-sequence voltage at the $k^{\text {th }}$ node could be solved by sensing the negative component of the grid voltage $v_{g \alpha+j \beta}^{-}$and computing $\left(i_{\alpha+j \beta}^{-}\right)_{i}$ for the current reference of each inverter to force $\left(v_{\alpha+j \beta}^{-}\right)_{k}=0$. However, $v_{g \alpha+j \beta}^{-}$is not available, and the computational load of the control scheme is lower if $G^{-}(s)_{i}(i=1 \ldots N)$ and $W^{-}(s)$ are not measured. Instead, a closed-loop system with a reference value equal to zero as input and with $\left(v_{\alpha+j \beta}^{-}\right)_{k}$ as a feedback signal is proposed. To distribute the inverters' effort in the form of negative-sequence current, a current sharing mechanism is proposed. Note that the summation term of (7) implies that infinite solutions exist for the inverters' currents to make $\left(v_{\alpha+j \beta}^{-}\right)_{k}$ zero. Thus, the current sharing feature imposes a single solution to the problem.

\section{CONTROL PROPOSAL}

\section{A. Control Scheme}

According to the previous discussion, two control objectives arise: first, negative-sequence voltage elimination at the $k^{\text {th }}$ node, and second, negative-sequence current sharing amongst the participating inverters. Fig. 2 depicts the negative-sequence control scheme to accomplish with the two control objectives. Local and remote signals are denoted by solid and dashed lines, respectively. The remote signals are transmitted by a Fast Ethernet network at a low transmission rate that inherently induces variable time delays of the order of microseconds. The standard [24] is taken as a reference to choose a transmission rate of $10 \mathrm{~Hz}$. Thus, making the impact of the Ethernet communication delays negligible. See [25] and [26] for recent literature reviews of communications and microgrid control, and [27] for an accurate theoretical analysis of the trade-off between transmission rates and time-delays in generic networked control systems. Future work could consider the impact of communication delays and the 
robustness against faults/failures in the communication link. The input signals for the $i^{\text {th }}$ controller are: the voltage reference $\left(v_{\alpha+j \beta}^{-}\right)^{*}$, the measured voltage unbalance at the $k^{\text {th }}$ node $\left(v_{e}^{-} \alpha+j \beta\right)_{k}$ and the reference currents of the other controller counterparts $\left(i_{\alpha+j \beta}^{-}\right)_{h}^{*} \forall h \neq i(h=1 . . N)$ for current sharing control. The voltage $\left(v_{e}^{-} \alpha+j \beta\right)_{k}$ is obtained from a remote unit that measures the three-phase voltage at the $k^{\text {th }}$ node, transforms it into the $\alpha \beta$ stationary frame and extracts the negative-sequence symmetrical component using a sequence extractor based on a second order generalized integrator (SOGI) [28], [29]. The $i^{\text {th }}$ inverter internal currentloop perfectly tracks the controller current reference output $\left(i_{\alpha+j \beta}^{-}\right)_{i}^{*}$ and injects its contribution of negative-sequence current $\left(i_{\alpha+j \beta}^{-}\right)_{i}$ into the power network to eliminate the voltage unbalance at the $k^{\text {th }}$ node. The internal current-loop is also connected to the standard active power control scheme [12], not depicted in Fig. 2, that provides the $i^{\text {th }}$ positivesequence current command $\left(i_{\alpha+j \beta}^{+}\right)_{i}^{*}$ given by

$$
\left(i_{\alpha+j \beta}^{+}\right)_{i}^{*}=\frac{2}{3} \frac{\left(v_{\alpha+j \beta}^{+}\right)_{i}}{\left(V^{+}\right)_{i}^{2}} P_{i}^{*}
$$

with $\left(v_{\alpha+j \beta}^{+}\right)_{i}$ and $\left(V^{+}\right)_{i}$ the positive-sequence voltage at the $i^{\text {th }}$ inverter output in its $\alpha \beta$ complex form and its amplitude, respectively, and $P_{i}^{*}$ the $i^{\text {th }}$ active power reference. It is important to observe that the proposed control scheme partially follows a centralized master/slave paradigm. Its operation heavily relies on the remote unit that measures the voltage being sent to all the controllers. Being this measurement unit a single point of failure, reliability could be ensured by introducing redundant equipment in the measurement point. Furthermore, high overall system reliability would also demand a redundant communication link. See [26] for a discussion on the reliability and resilience of microgrids.

\section{B. Negative-Sequence Controller}

The $i^{t h}$ negative-sequence controller time domain equation proposed in this work is given by

$$
\begin{gathered}
\left(i_{\alpha+j \beta}^{-}\right)_{i}^{*}=k_{v} e^{-j \omega_{0} t} \int e^{j \omega_{0} t}\left(\left(v_{\alpha+j \beta}^{-}\right)^{*}-\left(v_{e \alpha+j \beta}^{-}\right)_{k}\right) d t \\
+k_{c} e^{-j \omega_{0} t} \int e^{j \omega_{0} t}\left(k_{w i}\left(i_{\alpha+j \beta}^{-}\right)_{a v g}^{*}-\left(i_{\alpha+j \beta}^{-}\right)_{i}^{*}\right) d t
\end{gathered}
$$

with

$$
\left(i_{\alpha+j \beta}^{-}\right)_{a v g}^{*}=\frac{\sum_{i=1}^{N}\left(i_{\alpha+j \beta}^{-}\right)_{i}^{*}}{N} .
$$

It consists of two negative-sequence synchronous integrators in the $\alpha \beta$ stationary frame. The first one will assure eliminating the voltage unbalance at the $k^{\text {th }}$ node. The second one will distribute the necessary negative-sequence current amongst the participant inverters. The $i^{t h}$ weighting factor $k_{w i}$ allows adjusting the negative-sequence current contribution of each inverter. This is useful to cope with different inverters' capabilities or to reduce the inverters' negative-sequence voltages, as discussed in detail in subsection V. C. The local frequency locked loop in each inverter measures the grid angular frequency $\omega_{0}$. The term $e^{j \omega_{0} t}$ transforms the error signal to the synchronous reference frame, while $e^{-j \omega_{0} t}$ transforms the integrator result to the $\alpha \beta$ stationary frame. The complex control parameters $k_{v}=k_{v r}+j k_{v i}$ and $k_{c}=$ $k_{c r}+j k_{c i}$ are designed for setting the dynamics of the voltage unbalance elimination and the collaborative current sharing, respectively.

\section{C.Negative-Sequence Controller Transfer Function}

Since the $i^{\text {th }}$ negative-sequence controller output is the $i^{\text {th }}$ negative-sequence current reference (see Fig. 2) and realizing that $\left(i_{\alpha+j \beta}^{-}\right)_{a v g}^{*}$ contains $\left(i_{\alpha+j \beta}^{-}\right)_{i}^{*}$, the negative-sequence current error in the second integral term of (9) can be expressed as

$$
\begin{gathered}
k_{w i}\left(i_{\alpha+j \beta}^{-}\right)_{a v g}^{*}-\left(i_{\alpha+j \beta}^{-}\right)_{i}^{*}= \\
k_{w i} \frac{\sum_{h=1 h \neq i}^{N}\left(i_{\alpha+j \beta}^{-}\right)_{h}^{*}}{N}-\left(1-\frac{k_{w i}}{N}\right)\left(i_{\alpha+j \beta}^{-}\right)_{i}^{*} .
\end{gathered}
$$

Replacing the second term of (11) in (9) and assuming that the grid frequency $\omega_{0}$ is constant, the controller transfer function is obtained from the time derivate of (9), which, after some rearrangements, can be expressed as

$$
\begin{aligned}
& \frac{d\left(i_{\alpha+j \beta}^{-}\right)_{i}^{*}}{d t}=-j \omega_{0}\left(i_{\alpha+j \beta}^{-}\right)_{i}^{*}+k_{v}\left(\left(v_{\alpha+j \beta}^{-}\right)^{*}-\left(v_{e \alpha+j \beta}^{-}\right)_{k}\right) \\
& +k_{c}\left(k_{w i} \frac{\sum_{h=1 h \neq i}^{N}\left(i_{\alpha+j \beta}^{-}\right)_{h}^{*}}{N}-\left(1-\frac{k_{w i}}{N}\right)\left(i_{\alpha+j \beta}^{-}\right)_{i}^{*}\right) .
\end{aligned}
$$

By expressing (12) in the Laplace domain and grouping the terms with the complex variable $\left(i_{\alpha+j \beta}^{-}\right)_{i}^{*}$, yields

$$
\begin{gathered}
\left(i_{\alpha+j \beta}^{-}\right)_{i}^{*}=C_{v}(s)_{i} \cdot\left(\left(v_{\alpha+j \beta}^{-}\right)^{*}-\left(v_{e \alpha+j \beta}^{-}\right)_{k}\right) \\
+C_{c}(s)_{i} \cdot \sum_{h=1}^{N}\left(i_{\alpha+j \beta}^{-}\right)_{h}^{*}
\end{gathered}
$$

with

$$
\begin{gathered}
C_{v}(s)_{i}=\frac{k_{v}}{s+j \omega_{0}+k_{c}\left(1-\frac{k_{w i}}{N}\right)} \\
C_{c}(s)_{i}=\frac{k_{w i} k_{c} / N}{s+j \omega_{0}+k_{c}\left(1-\frac{k_{w i}}{N}\right)} .
\end{gathered}
$$

The transfer functions (14) and (15) are for the negativesequence voltage elimination and for the collaborative currentsharing, respectively.

\section{D.Negative-Sequence Closed-loop System}

Fig. 3 shows the block diagram of the closed-loop system and the $i^{\text {th }}$ controller expressed by (13). As in Fig. 2, dashed lines denote signals received from or sent to other inverters and the remote voltage unbalance measuring unit. The $i^{t h}$ controller generates the current reference $\left(i_{\alpha+j \beta}^{-}\right)_{i}^{*}$ from $C_{v}(s)_{i}$ and $C_{c}(s)_{i}$ outputs. $C_{v}(s)_{i}$ processes the voltage error signal given by the difference between the reference input $\left(v_{\alpha+j \beta}^{-}\right)^{*}=0$ and the feedback signal $\left(v_{e \alpha+j \beta}^{-}\right)_{k}$. The latter is obtained from the remote sensing unit. The sensor transfer 


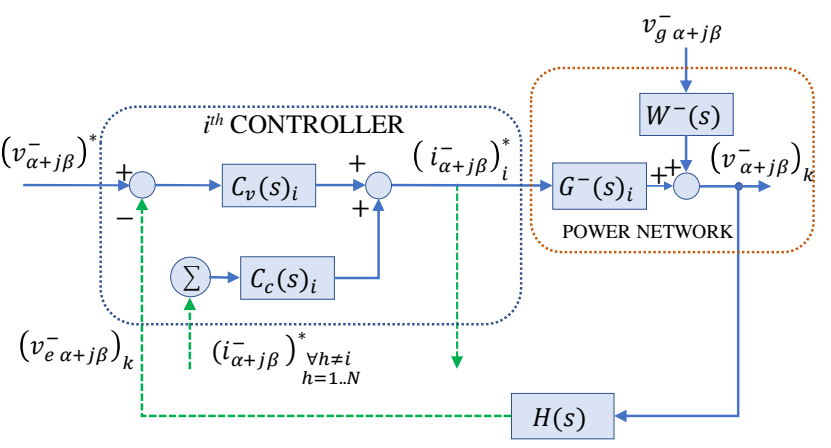

Fig. 3. Closed-loop system for the $i^{\text {th }}$ controller.

function $H(s)$ corresponds to the SOGI. Its model described by its complex form is [29]

$$
H(s)=\frac{\xi \omega_{0} s-j \xi \omega_{0}^{2}}{s^{2}+2 \xi \omega_{0} s+\omega_{0}^{2}}
$$

where $\xi$ is the damping factor. $C_{c}(s)_{i}$ takes the sum of the other counterparts' reference currents to balance $\left(i_{\alpha+j \beta}^{-}\right)_{i}^{*}$.

Considering $v_{g}^{-} \alpha+j \beta$ as a perturbation and assuming a controller design with slow dynamics, working with the closed-loop block diagram in Fig. 3, the following complex transfer functions are obtained

$$
\begin{gathered}
\frac{\left(v_{\alpha+j \beta}^{-}\right)_{k}}{\left(v_{\alpha+j \beta}^{-}\right)^{*}}=\frac{C_{v}(s)_{i} G^{-}(s)_{i}}{1+C_{v}(s)_{i} G^{-}(s)_{i} H(s)} \\
\frac{\left(v_{\alpha+j \beta}^{-}\right)_{k}}{\sum_{\substack{h=1 \\
h \neq i}}^{N}\left(i_{\alpha+j \beta}^{-}\right)_{h}^{*}}=\frac{C_{c}(s)_{i} G^{-}(s)_{i}}{1+C_{v}(s)_{i} G^{-}(s)_{i} H(s)} \\
\frac{\left(i_{\alpha+j \beta}^{-}\right)_{i}^{*}}{\sum_{\substack{N=1 \\
h \neq i}}^{N}\left(i_{\alpha+j \beta}^{-}\right)_{h}^{*}}=\frac{C_{c}(s)_{i}}{1+C_{v}(s)_{i} G^{-}(s)_{i} H(s)} .
\end{gathered}
$$

(17) is the input reference to output voltage transfer function. (18) and (19) are the sum of current references from the other counterparts to negative-sequence voltage and $i^{\text {th }}$ current reference, respectively. For complex transfer functions, the frequency response is not necessarily the conjugate symmetric for positive and negative frequencies [30], [31]. This fact allows designing a complex negative-sequence controller that has deep effects at a specific frequency $\left(s=-j \omega_{0}\right)$ and almost no effect at its conjugate $\left(s=j \omega_{0}\right)$. For this reason, the operation of positive and negative-sequence controllers is considered decoupled. As a result, there will be no interaction with the positive-sequence controller that controls the active power injection.

\section{STABILITY AND CONTROLLER DESIGN}

The goal is to eliminate the voltage unbalance at the node of interest collaboratively. First, the model of the AC microgrid setup is presented to derive the plant model expression for the node of interest. Second, applying the Generalized RouthHurwitz (GRH) criterion [32] to the characteristic polynomial

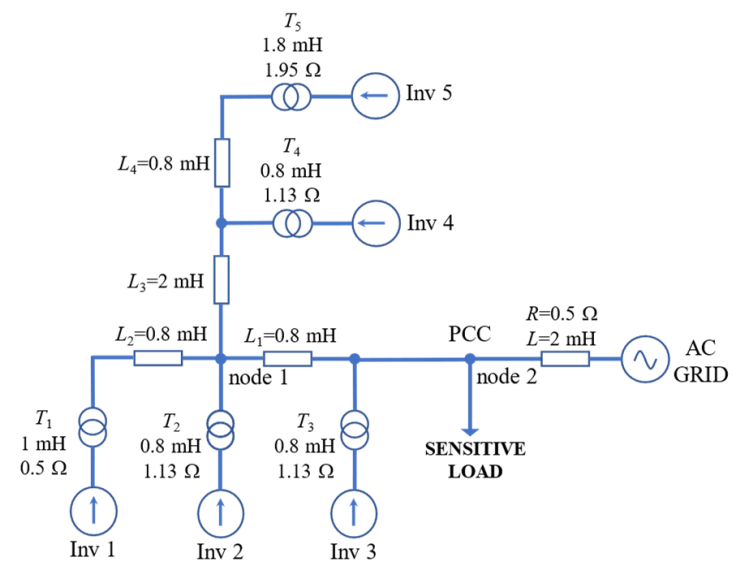

Fig. 4. Grid-connected AC microgrid setup.

with complex coefficients of (17), the stability condition for the negative-sequence voltage complex control parameter $k_{v}=k_{v r}+j k_{v i} \quad$ is obtained. Third, time-response specifications to the transient response are used for its design. Both the stability analysis and the design of $k_{v}$ are approached considering that only one inverter takes over the task of eliminating the voltage unbalance at the node of interest, with its negative-sequence controller in operation. Fourth, the current sharing control parameter $k_{c}=k_{c r}+j k_{c i}$ is designed under the premise that the negative-sequence voltage control loop has reached the setpoint. Fifth, the steady-state condition is applied to design the weighting factor and to obtain the steady-error of the negative-sequence voltage loop. Finally, a sensitivity analysis is performed to determine the robustness of the control proposal to the variations of the plant.

\section{A. Model of the AC Microgrid Setup}

Fig.4 depicts the AC microgrid setup as the power network configuration chosen for this work. The setup consists of five inverters (Inv1 to Inv5) connected to power transformers $\left(T_{1}\right.$ to $T_{5}$ ), each transformer is modeled with its characteristic impedance. Nodes are interconnected through inductive line impedances ( $L_{1}$ to $\left.L_{4}\right)$. The connection of the main grid to the AC microgrid is through an $R$ - $L$ line. The sensitive load, formed by a series $R_{L} L_{L}$ branch connected in parallel with a capacitor $C_{L}$, is located at node 2 . Node 2 location coincides with the PCC and it is selected for defining the plant model expression. Node 1 will be treated in the experimental part to show that the control proposal can eliminate the voltage unbalance at any node of the microgrid.

Applying (7) to node $2(k=2)$,

$\left(v_{\alpha+j \beta}^{-}\right)_{2}=\sum_{i=1}^{5} G^{-}(s)_{i}\left(i_{\alpha+j \beta}^{-}\right)_{i}+W^{-}(s) v_{g}^{-} \alpha+j \beta$

and using MNA, the customized expressions of $G^{-}(s)_{i} \quad(i=$ $1 . .5)$ and $W^{-}(s)$ for node 2 are obtained

$$
\begin{gathered}
G^{-}(s)_{i}=\frac{L_{L} L s^{2}+\left(L_{L} R+R_{L} L\right) s+R_{L} R}{B(s)} \forall i \\
W^{-}(s)=\frac{L_{L} s+R_{L}}{B(s)}
\end{gathered}
$$




$$
\begin{gathered}
B(s)=C_{L} L_{L} L s^{3}+\left(C_{L} L_{L} R+C_{L} R_{L} L\right) s^{2}+\left(C_{L} R_{L} R\right. \\
\left.+L+L_{L}\right) s+R_{L}+R
\end{gathered}
$$

Usually, in the frequency range of interest, the load presents a much higher impedance than the line impedance [18]. As an example, being the values of the plant $\left(R_{L}=24 \Omega\right.$, $\left.L_{L}=30 \mathrm{mH}, C_{L}=22 \mu \mathrm{F}, L=2 \mathrm{mH}, R=0.5 \Omega\right)$, (21) and (22) can be approximated in the frequency range of interest as

$$
\begin{gathered}
G^{-}(s)_{i}=L s+R \quad \forall i(i=1 . .5) \\
W^{-}(s)=1 .
\end{gathered}
$$

\section{B. Stability Analysis}

The stability analysis and the design of $k_{v}$ is carried out with only one inverter, $\left(N=1\right.$ and $\left.\sum_{h=1 h \neq i}^{N}\left(i_{\alpha+j \beta}^{-}\right)_{h}^{*}=0\right)$, and disabling the collaborative feature, $\left(k_{c}=0\right.$ and $\left.k_{w i}=0\right)$. First, the characteristic polynomial is obtained by inserting (14) - (16) and (23) in (17)

$$
\begin{gathered}
q(s)=c_{3} s^{3}+\left(c_{2}+j d_{2}\right) s^{2}+\left(c_{1}+j d_{1}\right) s+\left(c_{0}+j d_{0}\right) \\
c_{3}=1 \\
c_{2}=2 \xi \omega_{0}+L k_{v r} \xi \omega_{0} \\
d_{2}=\omega_{0}+L k_{v i} \xi \omega_{0} \\
c_{1}=\omega_{0}^{2}+R k_{v r} \xi \omega_{0}+L k_{v i} \xi \omega_{0}^{2} \\
d_{1}=2 \xi \omega_{0}^{2}+R k_{v i} \xi \omega_{0}-L k_{v r} \xi \omega_{0}^{2} \\
c_{0}=R k_{v i} \xi \omega_{0}^{2} \\
d_{0}=\omega_{0}^{3}-R k_{v r} \xi \omega_{0}^{2} .
\end{gathered}
$$

Second, and according to GRH criterion, being $q(\mathrm{~s})$ a cubic polynomial, the roots of (25) are in the left-half plane, only if the determinants $\Delta_{r}>0(r=1,2,3)$, i.e. the poles of (17) have negative real part. In this case the system is stable [32].

$$
\begin{gathered}
\Delta_{1}=c_{2} \\
\Delta_{2}=\operatorname{det}\left(\begin{array}{cccc}
c_{2} & c_{0} & -d_{1} \\
c_{3} & c_{1} & -d_{2} \\
0 & d_{1} & c_{2}
\end{array}\right) \\
\Delta_{3}=\operatorname{det}\left(\begin{array}{ccccc}
c_{2} & c_{0} & 0 & -d_{1} & 0 \\
c_{3} & c_{1} & 0 & -d_{2} & -d_{0} \\
0 & c_{2} & c_{0} & 0 & -d_{1} \\
0 & d_{1} & 0 & c_{2} & c_{0} \\
0 & d_{2} & d_{0} & c_{3} & c_{1}
\end{array}\right) .
\end{gathered}
$$

\section{C.Design of $k_{v}$}

Replacing the numerical values $L=2 \mathrm{mH}, R=0.5 \Omega$, $\xi=0.78$ and $\omega_{0}=2 \pi 60 \mathrm{rad} / \mathrm{s}$ in (26) and then solving $\Delta_{r}>0$ $(r=1,2,3)$, the limit values of $k_{v r}$ and $k_{v i}$ for stability are obtained

$$
0 \leq k_{v r} \leq 947.45 \text { and } k_{v i} \geq 0 .
$$

The suitable values of the control parameters $k_{v r}$ and $k_{v i}$ within the intervals defined by (30) are designed by pole placement. Thus, time-response specifications for the negative-sequence voltage elimination can be derived from the location of the closed-loop dominant pole. Since (25) presents complex coefficients and the system under study has been defined using complex variables, the complex poles do not have to appear necessarily as complex conjugates [33]. The system has a dominant low-frequency pole and two highfrequency poles. Then, the dynamics of the system is dominated by a single complex (low-frequency) pole. During transients, the settling-time of the modulus and the timederivative of the argument, i.e. angular frequency, of the complex output variable under control $\left(v_{\alpha+j \beta}^{-}\right)_{2}$, depend on the real and imaginary part of the dominant pole $\left(-\sigma+j \omega_{d}\right)$, respectively.

Referring to the real part design, the feedback signal $\left(v_{e}^{-} \alpha+j \beta\right)_{2}$ is sent by the measuring remote unit at a transmission frequency of $10 \mathrm{~Hz}$. Hence, the voltage-loop time constant must be dimensioned for at least twice as large as the transmission rate. Thus, fixing $\sigma=2$, the settling time is

$$
t_{s}=\frac{4}{\sigma}=2 \mathrm{~s}(2 \% \text { criterion })
$$

which leads to a voltage-loop time constant of

$$
\tau=\frac{t_{s}}{4}=0.5 \mathrm{~s}
$$

more than enough for the low transmission rate requirement.

The imaginary part is set at $-\omega_{o}=-2 \pi 60 \mathrm{rad} / \mathrm{s}$ to avoid interfering with the sequence extractor and with the transformation term $e^{j \omega_{0} t}$ of (9) during the negative-sequence voltage elimination transient. Thus, the current reference will present a smooth response. Next, the values of the control parameters $k_{v r}$ and $k_{v i}$ are derived.

Fig. 5 shows the location of the dominant closed-loop pole varying $0 \leq k_{v r} \leq 5$ and $0 \leq k_{v i} \leq 5$. The different colors are used to clarify the design of $k_{v r}$ and $k_{v i}$ graphically. The two arrows show the pole movement when incrementing $k_{v r}$ and $k_{v i}$ with a step size of 0.2 from the initial position $(0-$ $j 377)$. To place the pole at $(-2-j 377)$, the values for $k_{v r}$ and $k_{v i}$ are obtained graphically resulting in $k_{v r}=1.2$ and $k_{v i}=1.8$. Note that for node $2, G^{-}(s)_{i}=L s+R \quad \forall i(i=$ 1..5), therefore, the designed values of $k_{v r}$ and $k_{v i}$ are the same for all the controllers.

Although the procedure shown for the design of $k_{v}$ is only valid for one inverter, it provides a reliable value of $k_{v}$ in the case that more than one inverter is enabled for the task of eliminating the voltage unbalance simultaneously. The combined action of all the negative-sequence voltage control loops working together provides a much faster equivalent dynamics. Consequently, to get an approximation of the original time-response specifications, $k_{v}$ should be reduced to $k_{v} / N$, being $N$ the number of inverters.

\section{D.Design of $k_{c}$}

Observing the controller denominator of (14) and (15), $k_{c}=$ $k_{c r}+j k_{c i}$ has the effect of moving the position of the dominant pole previously placed. On the one hand, values of $k_{c r}>0$ displace the pole horizontally to the left with an offset given by $k_{c r}\left(1-\frac{k_{w i}}{N}\right)$. On the other hand, $k_{c i}$ shifts the pole vertically moving the pole away from the $-377 \mathrm{rad} / \mathrm{s}$ goal. 


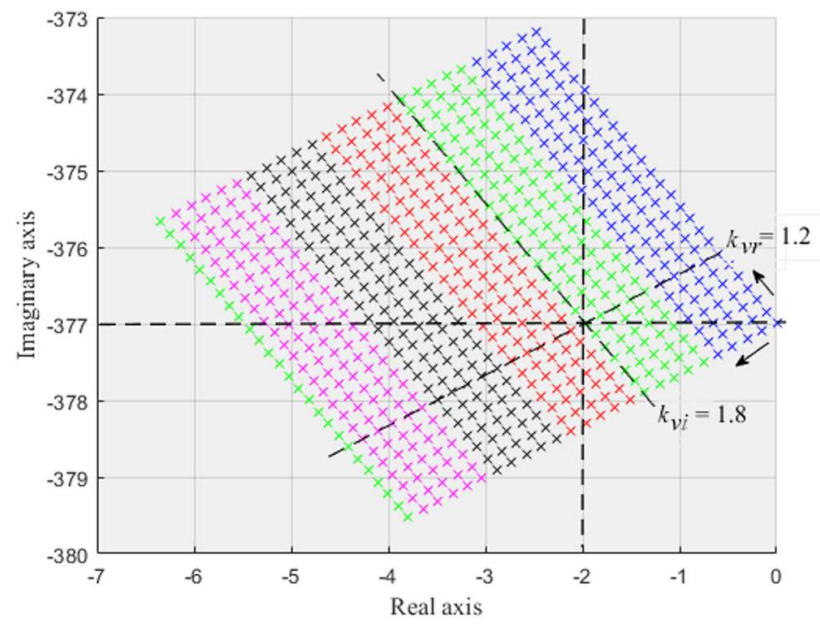

Fig. 5 Location of the dominant closed-loop pole varying $k_{v r}$ and $k_{v i}$.

Therefore, fixing $k_{c i}=0$ will keep the imaginary part of the dominant pole at $-377 \mathrm{rad} / \mathrm{s}$.

The control parameter $k_{c r}$ is designed considering that the system has reached the setpoint and it is in equilibrium, that is the negative-sequence voltage at the node of interest is zero. Then, there are an infinite number of solutions for $\left(i_{\alpha+j \beta}^{-}\right)_{i} \forall i(i=1 . . N)$ that keep $\left(v_{e}^{-} \alpha+j \beta\right)_{2}=0$. The system moves along the manifold associated with $\left(v_{e}^{-} \alpha+j \beta\right)_{2}=0$ freely, with the only restriction imposed by the total requested negative-sequence current necessary to eliminate the negativesequence voltage. Besides, the equivalent dynamics of all the participants is much faster due to the combined action of all the negative-sequence voltage control loops working together. Accordingly, to mitigate interactions between the two controls, the dynamics of the current sharing control must be slower than the negative-sequence voltage control.

Then, forcing $k_{c i}=0$, assuming that $\left(v_{e}^{-} \alpha+j \beta\right)_{2}$ has reached the reference and observing Fig. 3 and (15) the system reduces to

$$
\frac{\left(i_{\alpha+j \beta}^{-}\right)_{i}^{*}}{\sum_{\substack{h=1 \\ h \neq i}}^{N}\left(i_{\alpha+j \beta}^{-}\right)_{h}^{*}}=\frac{k_{w i} k_{c r} / N}{s+j \omega_{0}+k_{c r}\left(1-\frac{k_{w i}}{N}\right)} .
$$

Since

$$
\sum_{h=1 h \neq i}^{N}\left(i_{\alpha+j \beta}^{-}\right)_{h}^{*}=\left[\sum_{i=1}^{N}\left(i_{\alpha+j \beta}^{-}\right)_{i}^{*}\right]-\left(i_{\alpha+j \beta}^{-}\right)_{i}^{*}
$$

rearranging (33) leads to

$$
\left(i_{\alpha+j \beta}^{-}\right)_{i}^{*}=k_{w i}\left(i_{\alpha+j \beta}^{-}\right)_{\text {avg }}^{*} \frac{k_{c r}}{s+j \omega_{0}+k_{c r}} .
$$

The control parameter $k_{c r}$ can be used to set the settling time $t_{s}$ for reaching the $98 \%$ of the average of the reference currents times the $i^{t h}$ weighting factor. A good trade-off design for $k_{c r}$ is based on choosing $t_{s}=5 \mathrm{~s}$. In this case, the interactions of the voltage and current control loops are sufficiently reduced while maintaining an acceptable dynamics for the current control loop.

$$
\frac{1}{k_{c r}}=\tau=\frac{t_{s}}{4} \rightarrow k_{c r}=0.8
$$

Note that (35) does not depend on $G^{-}(s)_{i}$, therefore, the designed value of $k_{c r}$ is the same for all the controllers.

\section{E. Design of $k_{w i}$ and Steady-State Solution}

From (35), the negative-sequence reference current in steady-state $\left(s=-j \omega_{0}\right)$ can be written as

$$
\left(i_{\alpha+j \beta}^{-}\right)_{i}^{*}=k_{w i}\left(i_{\alpha+j \beta}^{-}\right)_{a v g}^{*}
$$

The design condition for $k_{w i}$ is derived by replacing (10) in (37) $\forall i(i=1 . . N)$ :

$$
\sum_{i=1}^{N} k_{w i}=N
$$

This condition is necessary for the right operation of the system, but it allows enough freedom to choose the contribution of each inverter to the negative-sequence current sharing.

If the $N$ inverters have reached $\left(i_{\alpha+j \beta}^{-}\right)_{a v g}^{*}$, then (11) can be written as

$$
\sum_{h=1 h \neq i}^{N}\left(i_{\alpha+j \beta}^{-}\right)_{h}^{*}=\left(N / k_{w i}-1\right)\left(i_{\alpha+j \beta}^{-}\right)_{i}^{*} .
$$

The closed-loop diagram in Fig. 3 can be rearranged so that the input of $C_{c}(s)_{i}$ is (39). Working with the diagram and simplifying, (17) turns into

$$
\frac{\left(v_{\alpha+j \beta}^{-}\right)_{k}}{\left(v_{\alpha+j \beta}^{-}\right)^{*}}=\frac{k_{v} G^{-}(s)_{i}}{\left(s+j \omega_{0}\right)+k_{v} G^{-}(s)_{i} H(s)} .
$$

At $s=-j \omega_{0}=-j 2 \pi 60 \mathrm{rad} / \mathrm{s}$, the sequence extractor $H(s)$ presents a unitary gain and a phase of $0^{\circ}$, and $\left(s+j \omega_{0}\right)=0$. Thus, (40) presents a unitary gain, i.e. no steady-state error will be noticed.

\section{F. Sensitivity Analysis}

The sensitivity analysis to determine how variations of the plant $G^{-}(s)_{i}$ affect stability is shown next. For the closed-loop system of Fig. 3, the function

$$
D(s)=1+C_{v}(s)_{i} G^{-}(s)_{i} H(s)
$$

represents the distance of the open-loop transfer function $C_{v}(s)_{i} G^{-}(s)_{i} H(s)$ to the critical point $(-1+j 0)$ at each frequency $s=j \omega$ in the Nyquist diagram [34]. Forcing the open-loop transfer function to cross the critical point, i.e. distance equals zero, yields

$$
\begin{gathered}
D(s)=1+C_{v}(s)_{i} G^{-}(s)_{i} H(s)=0 \\
G^{-}(s)_{i}=-\frac{1}{C_{v}(s)_{i} H(s)} .
\end{gathered}
$$

Inserting (23), (14) and (16), replacing $s=j \omega$ and separating real and imaginary parts in (43), the pairs of values of $R$ and $L$ that cause instability making the Nyquist curve cross the critical point as a function of the frequency $\omega$ are obtained 


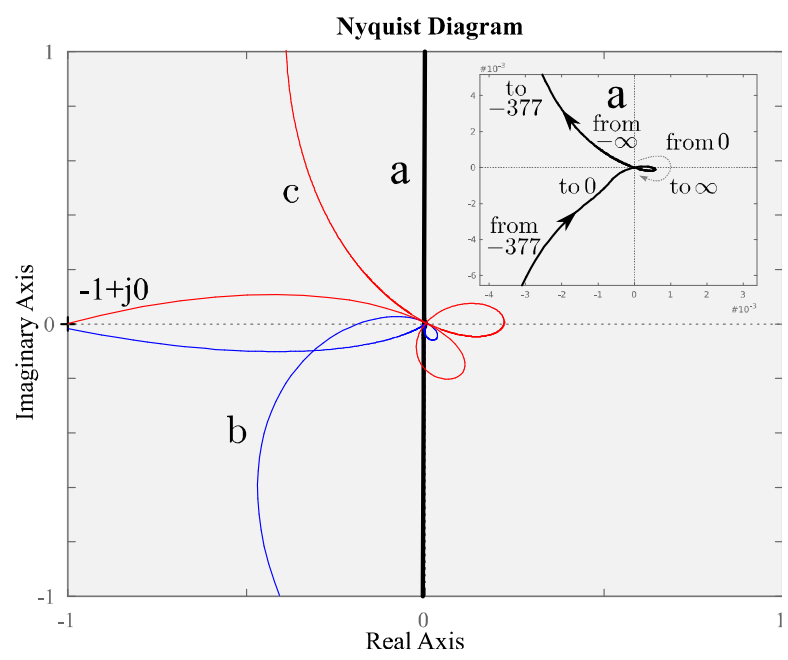

(a)
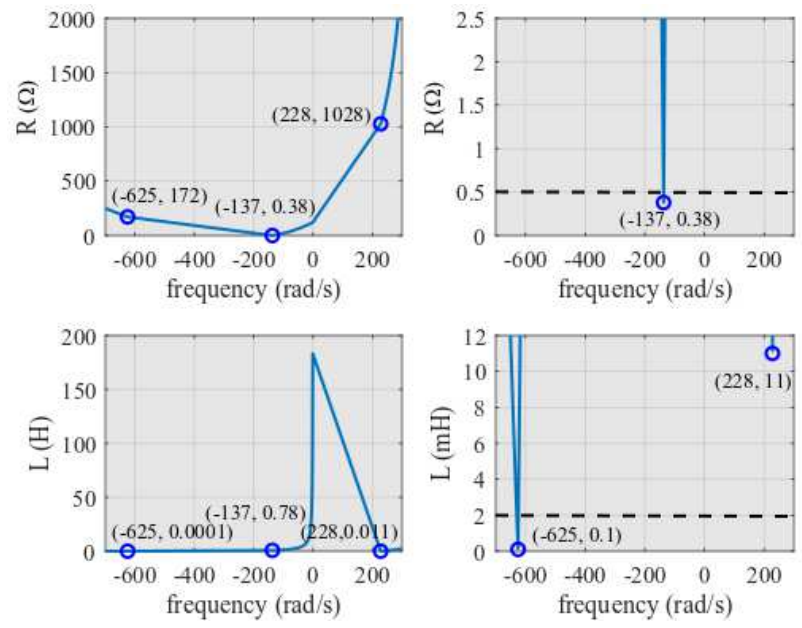

(b)

Fig. 6. (a) Loop transfer function $C_{v}(s)_{i} G^{-}(s)_{i} H(s)$ for different pairs of values of $R$ and $L$. (b) Pairs of $R$ and $L$ values for instability vs frequency.

$$
\begin{aligned}
R & =\frac{2 k_{v i} \xi \omega \omega_{0}\left(\omega+\omega_{0}\right)-k_{v r}\left(\omega+\omega_{0}\right)\left(\omega^{2}-\omega_{0}^{2}\right)}{\xi \omega_{0}\left(\omega_{0}-\omega\right)\left({k_{v i}}^{2}+{k_{v r}}^{2}\right)} \\
L & =\frac{2 k_{v r} \xi \omega \omega_{0}\left(\omega+\omega_{0}\right)+k_{v i}\left(\omega+\omega_{0}\right)\left(\omega^{2}-\omega_{0}^{2}\right)}{\omega \xi \omega_{0}\left(\omega_{0}-\omega\right)\left({k_{v i}}^{2}+{k_{v r}}^{2}\right)}
\end{aligned}
$$

Fig. 6 (a) depicts the Nyquist diagram of the loop-gain transfer function $C_{v}(s)_{i} G^{-}(s)_{i} H(s)$ for different pairs of values of $R$ and $L$. The solid black curve (a) is for the nominal values of the system $\left(L=2 \mathrm{mH}, R=0.5 \Omega, k_{v r}=1.2\right.$, $\left.k_{v i}=1.8, \xi=0.78, \omega_{0}=2 \pi 60 \mathrm{rad} / \mathrm{s}\right)$. Note that it lies practically on the imaginary axis. The plot on the upper righthand side zooms in curve (a) near the origin $(0+j 0)$. The gain is zero at $-\infty$ frequency, then it evolves to infinite gain at $\omega_{0}=-377 \mathrm{rad} / \mathrm{s}$. Finally, it passes through zero gain at zero and $+\infty$ frequencies. This curve is very far away from crossing the critical point $(-1+j 0)$ and confirms the stability design of subsection IV. B.

Next, instability is analyzed by introducing the nominal values $\left(k_{v r}=1.2, k_{v i}=1.8, \xi=0.78, \omega_{0}=2 \pi 60 \mathrm{rad} / \mathrm{s}\right)$ in (44) and (45). Fig. 6 (b), left side, shows the result of pairs of $R$ and $L$ that cause instability by sweeping $\omega$ from -10000 to $10000 \mathrm{rad} / \mathrm{s}$. The pairs $R$ and $L$ are both positive only in the range -700 to $300 \mathrm{rad} / \mathrm{s}$. Fig. 6(b), right side, vertically enlarges the results of the left side to focus only on practical values of $R$ and $L(R \leq 2.5 \Omega$ and $L \leq 12 \mathrm{mH})$. The dashed horizontal lines denote the nominal values $(R=0.5 \Omega$ and $L=2 \mathrm{mH}$ ). In these subfigures, three points are marked to lead the following discussion. In the first point, note that $L=$ $0.1 \mathrm{mH}$ at $\omega=-650 \mathrm{rad} / \mathrm{s}$, however, $R=172 \Omega$, which is clearly out of the plot limits. In the second point, $R=0.38 \Omega$ at $\omega=-137 \mathrm{rad} / \mathrm{s}$, but now $L=0.78 \mathrm{H}$, which largely surpasses the plot boundaries. In the third point, $L=11 \mathrm{mH}$ at $\omega=228 \mathrm{rad} / \mathrm{s}$, however $R=1028 \Omega$. From this discussion, it is clear that instability is only reached for impractical values of $R$ and $L$. Going back to Fig. 6 (a), the Nyquist curves for the two first points of the previous discussion are shown denoted as $b$ and $c$, respectively. Note that these curves cross the critical point, which validates the instability of the system for these impractical values of $R$ and $L$. As a final remark, it is worth mentioning that the system is stable for practical (realistic) values of $R$ and $L$.

\section{V.EXPERIMENTAL RESULTS}

The proposed control strategy has been tested in the laboratory setup depicted in Fig. 7. Each one of the five threephase inverters has a rated power of $3.3 \mathrm{kVA}$ at $110 \mathrm{~V}_{\mathrm{rms}}, 10$ $A_{\text {rms. }}$. The filter at the inverter output is LCL type. It is formed by a $5 \mathrm{mH}$ inductor at the inverter side, a $1.5 \mu \mathrm{F}$ capacitor with a series $68 \Omega$ damping resistor and the flux leakage inductor of the line transformer, see Fig. 4. The DC link voltage is $400 \mathrm{~V}$. The grid is emulated by a Pacific Power AC voltage source configured at $V_{a}, V_{b}, V_{c}=110,105,100 \mathrm{~V}_{\mathrm{rms}}$ respectively, at $60 \mathrm{~Hz}$. This is equivalent to a negative-sequence amplitude of $5 \mathrm{~V}$. This amplitude provides an unbalance factor of $3.2 \%$, above the recommended limit of $2 \%$ given by the EN50160 standard [10]. The sensitive load is purely resistive with a per phase value of $24 \Omega$.

Each inverter is managed by its own control board fitted with a Texas Instruments F28M36 Concerto dual core microcontroller. The active power injection control and the proposed controller depicted in Fig. 3 are implemented on each C28 core. The M3 communications core is for the lowbandwidth exchange of signals. All the control boards are connected to a hub card using an ethernet link. The sensing unit is implemented with the same control board. The internal current loop perfectly tracks the reference current supplied by the $i^{\text {th }}$ controller. A space vector modulator computes the switching times of each inverter leg. The signals of interest are exported to Matlab to represent the results. In this case, the negative-sequence voltage amplitude of $v_{\alpha+j \beta}^{-}$at the node of interest, the negative-sequence voltage amplitude at each inverter output and the negative-sequence reference current amplitude $\left(i_{\alpha+j \beta}^{-}\right)_{i}^{*}$ are considered for better showing the achievements. 


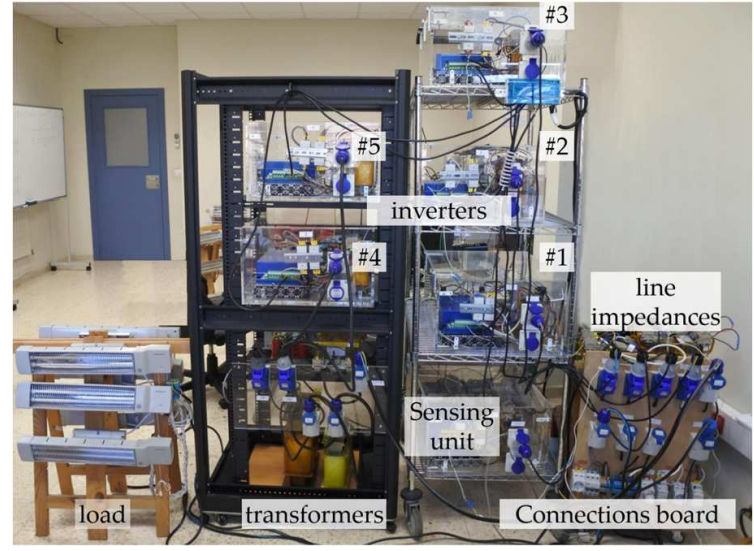

Fig. 7. Laboratory setup.

Four experiments have been performed. In all the experiments, the active power injection of each inverter is set to $200 \mathrm{~W}$. Experiment 1 tests the performance of the controller in terms of negative-sequence voltage elimination and the design of the control parameter $k_{v}$. As in subsections IV. B and $\mathrm{C}$, only one inverter (Inverter 1 ) is considered to eliminate the negative-sequence voltage at node 2 . Inverters 2 to 5 only inject active power and their respective negative-sequence controllers are disabled. In experiment 2, all the inverters participate in the negative-sequence voltage elimination at node 2 . The negative-sequence current sharing feature is evaluated according to the design of the control parameter $k_{c}$. The weighting factor $k_{w i}$ is adjusted for an even distribution of negative-sequence current. Experiment 3 is divided into two parts. The first one uses the weighting factor to reduce the contribution of the inverters with higher line impedance between its output and node 2 . The goal is to mitigate the negative-sequence output voltage of these inverters. The second one tests the controller performance under unbalanced load and line impedances with equal current sharing. Finally, experiment 4 validates the control proposal at node 1, see Fig. 4.

\section{A. Experiment 1}

In this experiment, only the negative-sequence controller of inverter 1 is enabled to eliminate negative-sequence voltage at node 2 . The negative-sequence controllers of inverters 2 to 5 are disabled and their negative-sequence currents are zero. Accordingly, the collaborative feature is disabled by making $k_{c r}=k_{c i}=0$. The five inverters are injecting active power from $t=0 \mathrm{~s}$. Fig. 8 (a) top shows the amplitude of the negative-sequence voltage $v_{\alpha+j \beta}^{-}$at node 2 with an initial value of $5 \mathrm{~V}$. The negative-sequence controller is activated in inverter 1 at $t=5 \mathrm{~s}$. The inverter injects the negativesequence current dictated by the controller reference $\left(i_{\alpha+j \beta}^{-}\right)_{1}^{*}$. Its amplitude is depicted in Fig. 8 (a) center. The negative-sequence voltage at each inverter output is shown in Fig. 8 (a) bottom. The control parameter $k_{v}$ is set according to the designed value in subsection IV. C $\left(k_{v r}=1.2\right.$ and $k_{v i}=$ 1.8). Observe that the controller eliminates the negativesequence voltage in the expected time of $2 \mathrm{~s}$. Moreover, the reference current exhibits a smooth response as projected. Conversely, in Fig 8 (b) center, the current reference shows an overshoot. In this case, the pole is displaced on purpose to $(-1-j 375.5)$ and using the graph in Fig. 5 yields $k_{v r}=2$ and $k_{v i}=0$. According to the new pole location, the negativesequence voltage goes to zero in $4 \mathrm{~s}$; see Fig. 8 (b) top.

As a result of inverter 1 injecting negative-sequence current, the negative-sequence voltage at the inverter output increases reaching a steady-state value of $7.5 \mathrm{~V}$ (VUF of $4.8 \%$ above the safe $2 \%$ limit). Besides, the inverter capability to inject active power is seriously reduced. The controller imposes a negativesequence reference current amplitude of $5 \mathrm{~A}=3.5 \mathrm{~A}_{\mathrm{rms}}$. This is $35 \%$ of the inverter rated current.

Although only inverter 1 is injecting negative-sequence current, the negative-sequence voltage at the output of inverters 2 to 5 is being reduced. Its value depends on the location of the inverter in the power network. Thus, inverter 3 is nearly zero because it is connected close to node 2 . Meanwhile, inverters 2, 4 and 5 share the same value because they see the negative-sequence voltage at node 1 .

\section{B. Experiment 2}

In this experiment, each negative-sequence controller of the five inverters is serially activated every $10 \mathrm{~s}$ from $t=5 \mathrm{~s}$, each one with the same setting of $k_{v r}=1.2$ and $k_{v i}=1.8$. The five inverters are injecting active power from $t=0 \mathrm{~s}$. Fig. 9 (a) illustrates the case of disabling the collaborative feature ( $k_{c r}=k_{c i}=0$ ), for sharing the negative-sequence current amongst the five inverters. Despite the controller can eliminate the negative-sequence voltage, Fig. 9 (a) top, neither the negative-sequence currents nor the negative-sequence voltages at each inverter output achieve a steady-state regime, Fig. 9 (a) center and bottom, respectively. Instead, they are oscillating. Observe that the experiment time has been extended to $180 \mathrm{~s}$ to appreciate a full oscillating period. As predicted in subsection IV. D, infinite solutions exist for each of the currents delivered by the inverters to eliminate the negativesequence voltage at the point of interest, and the currents are moving across these solutions due to a disturbance in the system (probably the noise).

Fig 9 (b) shows the result when activating the collaborative control with $k_{c r}=0.8$ and $k_{c i}=0$, as designed in subsection IV. D, and keeping $k_{v r}=1.2$ and $k_{v i}=1.8$. The goal is to control the response of the current sharing. For an even distribution of the negative-sequence current, the weighting factor $k_{w i}=1(i=1 \ldots 5)$. The negative-sequence voltage amplitude at node 2 is practically eliminated in 2 s, Fig. 9 (b) top. A remain of less than $0.4 \mathrm{~V}$ is noticed when inverter 2 is activated at $t=15 \mathrm{~s}$. However, it diminishes as more inverters are activated. Observing Fig. 9 (b) center, equal negative-sequence current sharing is achieved as soon as the next inverter is serially activated every $10 \mathrm{~s}$. According to the value of $k_{c r}$, the current achieves the steady-state value in $5 \mathrm{~s}$. It is worth highlighting that with this control proposal the effort in form of negative-sequence current is shared equally by each inverter. At $t>55 \mathrm{~s}$, all the inverters are operating with the negative-sequence current reference amplitude of 1 $A=0.7 \mathrm{~A}_{\text {rms }}$ ( $7 \%$ of the inverter rated current in front of $35 \%$ of experiment 1). This benefit is also translated into a reduction of negative-sequence voltage at each inverter output, Fig. 9 (b) bottom. Observe that as soon as inverter 2 is activated at $t=15 \mathrm{~s}$ the voltage is reduced from 7.5 to $2.5 \mathrm{~V}$. 

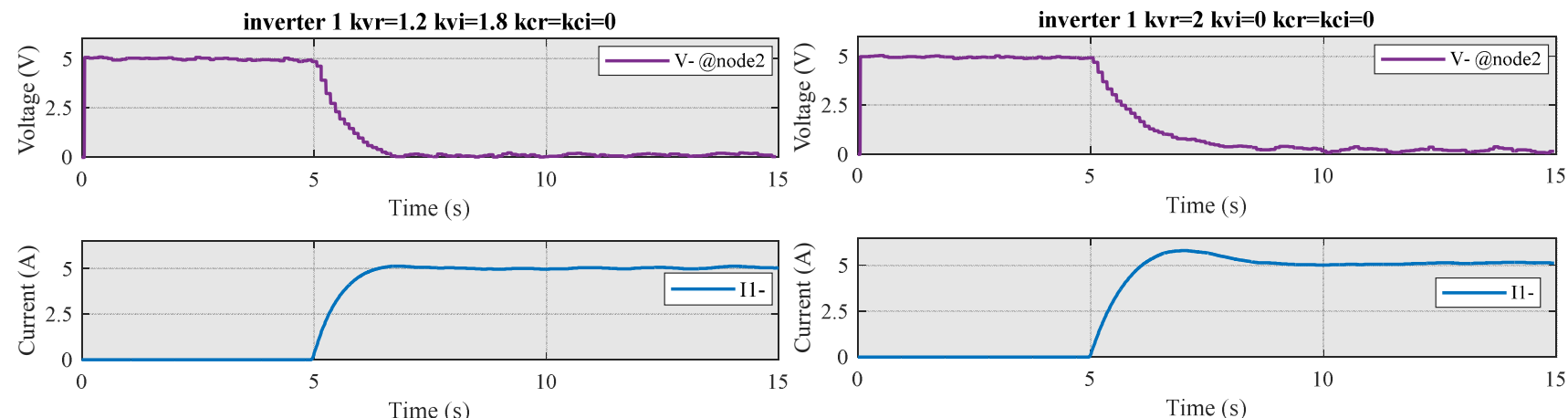

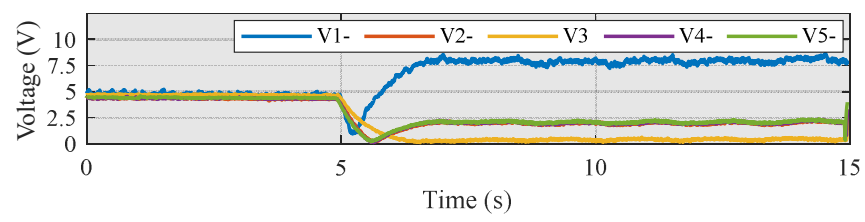

(a)

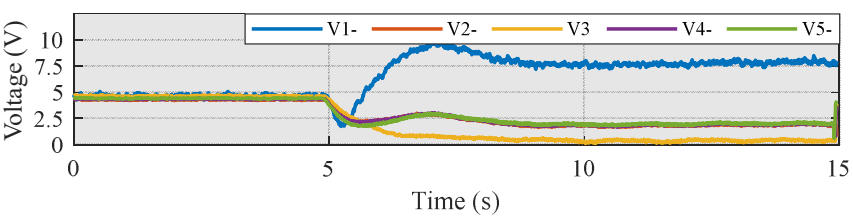

(b)

Fig. 8. Experiment 1. Top: amplitude of the negative-sequence voltage $V^{-}=\left|v_{\alpha+j \beta}^{-}\right|$at node 2. Center: amplitude of the negative-sequence reference current $I_{1}^{-}=\left|\left(i_{\alpha+j \beta}^{-}\right)_{1}^{*}\right|$ of inverter 1. Bottom: amplitude of the negative-sequence voltage at each inverter output. (a) Performance of the controller with designed control parameters. (b) Performance of the controller with new control parameters to get slower response in the negative-sequence voltage elimination and current overshoot.
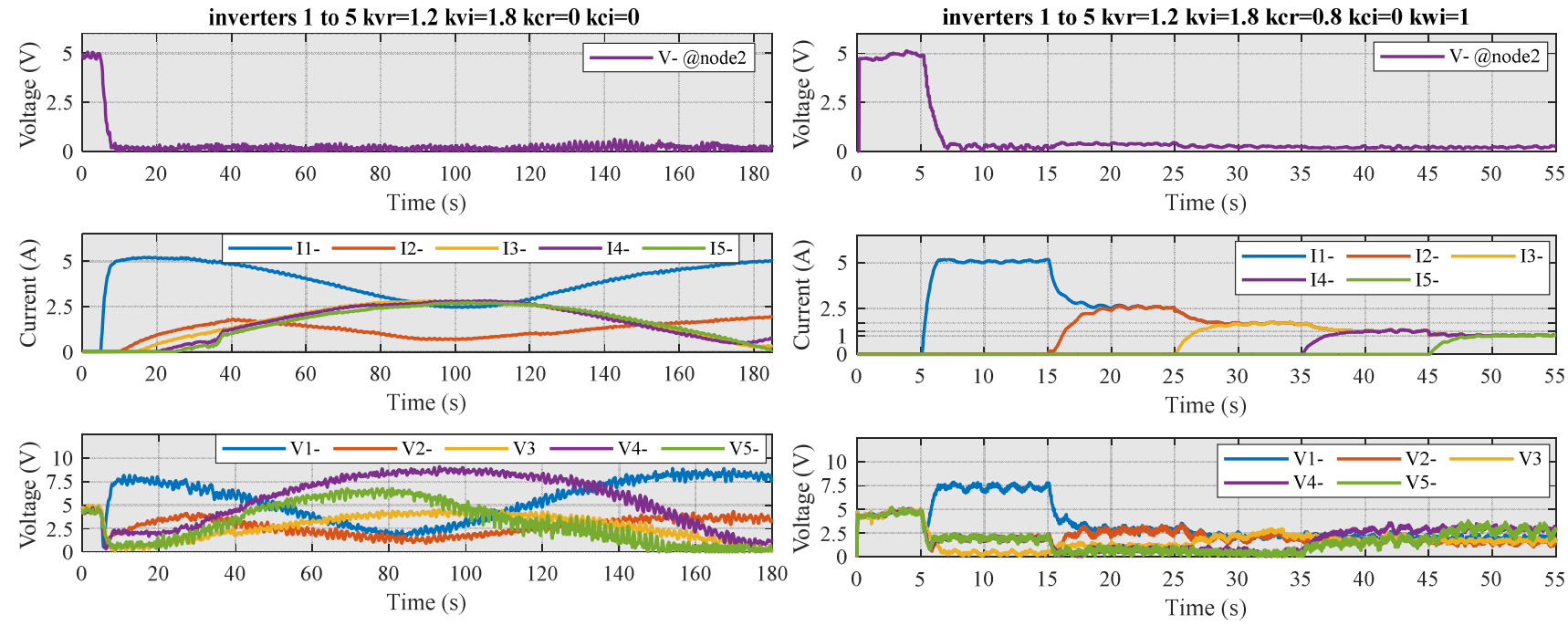

(a)

(b)

Fig. 9. Experiment 2. Top: amplitude of the negative-sequence voltage $V^{-}=\left|v_{\alpha+j \beta}^{-}\right|$at node 2. Center: amplitude of the negative-sequence reference current $I_{i}^{-}=\left|\left(i_{\alpha+j \beta}^{-}\right)_{i}^{*}\right|, i$ from 1 to 5. Bottom: amplitude of the negative-sequence voltage at each inverter output. Performance (a) disabling and (b) enabling current sharing feature with even distribution.

The steady-state negative-sequence voltage amplitudes for inverters 1 to 5 are $1.9,1.5,1.6,2.7,2.9 \mathrm{~V}$ respectively. The highest values correspond to the inverters 4 and 5. This is due to a higher impedance between its output and node 2, see Fig. 4. Inverter 5 presents a VUF $=1.9 \%$, below the recommended $2 \%$ limit though.

\section{Experiment 3}

In the first part of this experiment, the weighting factor is adjusted to reduce the contribution of the inverters 4 and 5 , $k_{w 1}=k_{w 2}=1, \quad k_{w 3}=2$ and $k_{w 4}=k_{w 5}=0.5$. The share of current not injected by inverters 4 and 5 is taken by inverter 3 . Thus, the negative-sequence output voltage of inverters 4 and 5 is alleviated. As in experiment 2 , the negative-sequence voltage is effectively eliminated, Fig. 10 (a) top. However, compared to experiment 2 , the new negative-sequence current distribution, Fig. 10 (a) center, reduces the negative-sequence output voltage of inverters 4 and 5, Fig. 10 (a) bottom at $t=$ $50 \mathrm{~s}$. Besides, when all the controllers are in operation, the negative-sequence output voltage of the inverters is centered around $1.5 \mathrm{~V}$ (VUF < 1\%). Fig. 10 (b) shows the second part of this experiment in which the controller performance is evaluated under both unbalanced load and line impedance with equal current sharing, $k_{w i}=1(i=1 \ldots 5)$. Keeping the voltage unbalance provided by the main grid, the load of phase $\mathrm{A}$ is disconnected and the line impedance between nodes 1 and 2 of phase $\mathrm{B}$ is short-circuited. Under these severe 

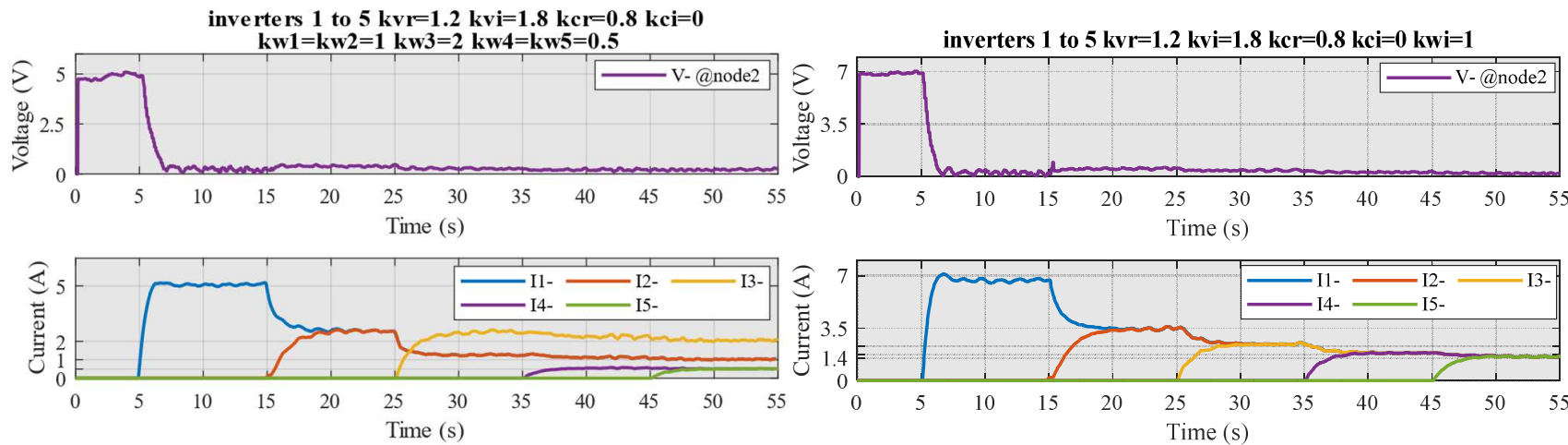

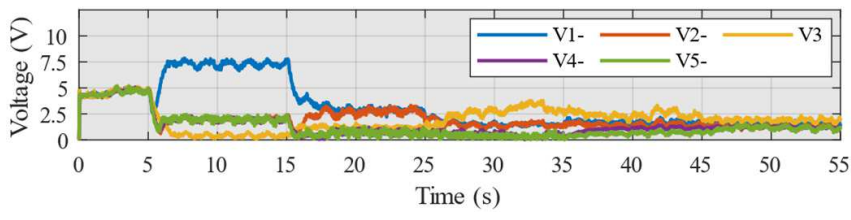

(a)

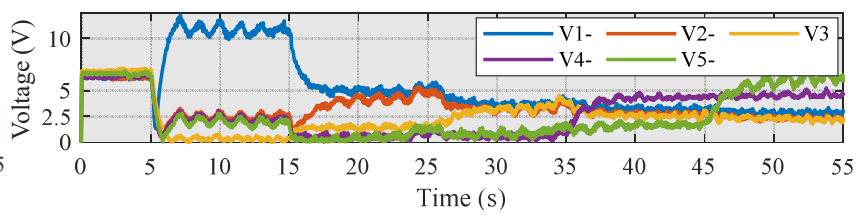

(b)

Fig. 10. Experiment 3. Top: amplitude of the negative-sequence voltage $V^{-}=\left|v_{\alpha+j \beta}^{-}\right|$at node 2 . Center: amplitude of the negative-sequence reference current $I_{i}^{-}=\left|\left(i_{\alpha+j \beta}^{-}\right)_{i}^{*}\right|, i$ from 1 to 5. Bottom: amplitude of the negative-sequence voltage at each inverter output. Performance (a) uneven current distribution and (b) unbalanced line and load impedances with even current distribution.
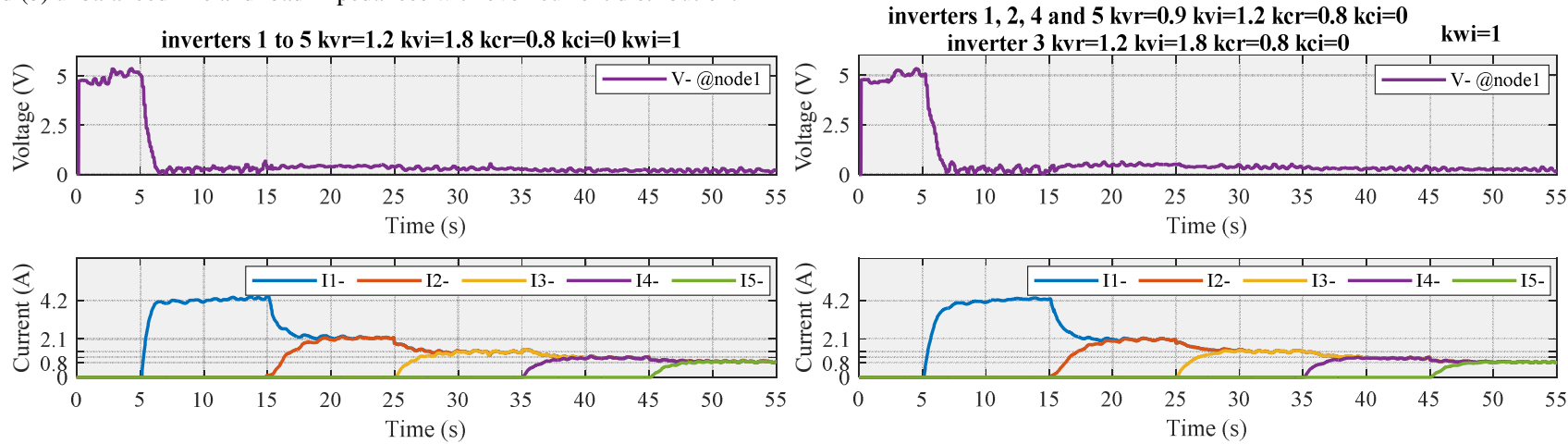

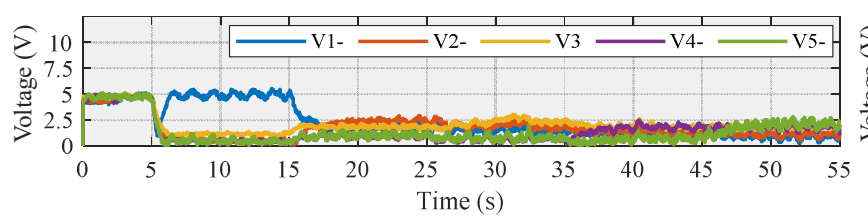

(a)

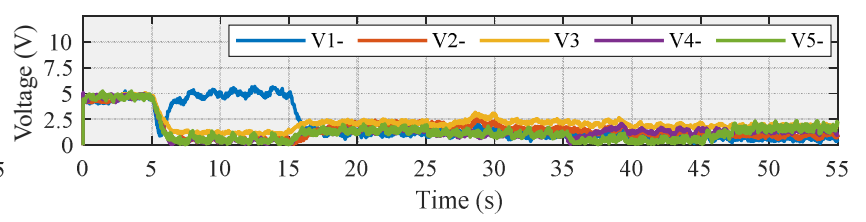

(b)

Fig. 11. Experiment 4. Top: amplitude of the negative-sequence voltage $V^{-}=\left|v_{\alpha+j \beta}^{-}\right|$at node 1 Center: amplitude of the negative-sequence reference current $I_{i}^{-}=\left|\left(i_{\alpha+j \beta}^{-}\right)_{i}^{*}\right|, i$ from 1 to 5 . Bottom: amplitude of the negative-sequence voltage at each inverter output Performance with even current distribution (a) keeping and (b) recomputing control parameters.

unbalanced conditions, the voltage unbalance at node 2 is of 7 $\mathrm{V}$ (VUF $=4.5 \%)$. The controller behaves effectively eliminating the voltage unbalance at node 2, Fig. 10 (b) top, injecting $7 \mathrm{~A}$. Due to the first integral term of the controller that forces the error signal to zero. Similarly, the current sharing operation gets unaffected, Fig. 10 (b) center. However, the impact of eliminating the voltage unbalance at node 2 on the voltage unbalance at the inverters' output is worse due to the higher current needed in this experiment, Fig. 10 (b) bottom. Observe that at $t=50 \mathrm{~s}$, the negative-sequence output voltage of inverter 5 is around $7 \mathrm{~V}(\mathrm{VUF}=4.5 \%)$, well above the recommended limit of $2 \%$.

\section{Experiment 4}

This experiment shows how the control proposal eliminates the negative-sequence voltage at any point of the $\mathrm{AC}$ microgrid. The point of interest is now node 1, see Fig. 4. Fig. 11 (a) illustrates the performance keeping the control parameters $k_{v r}, k_{v i}$ and $k_{c r}$ unchanged from the previous experiment at node 2. Alternatively, Fig. 11 (b) shows the performance recomputing the parameters following the design guidelines in Section IV. Using MNA at node $1, G^{-}(s)_{i}=$ $\left(L+L_{1}\right) s+R \quad \forall i(i=1,2,4,5) \quad$ and $\quad G^{-}(s)_{3}=L s+R$. Then, $k_{v}$ is recomputed for controllers $1,2,4$ and 5 to have the same dynamics, $k_{v r}=0.9$ and $k_{v i}=1$. Controller 3 remains the same. Note that little differences are appreciated. 
A slightly faster response on both the negative-sequence voltage elimination and current is noticed in Fig. 11 (a) when the control parameters are not recomputed despite the inductance has a $40 \%$ increase. The controller presents immunity against $R$ and $L$ variations, as announced in subsection IV. F. The current sharing feature follows the same dynamics in both Fig. 11 (a) and (b).

\section{CONCLUSION}

This paper presented a novel control scheme in which distributed grid-feeding inverters collaborate to eliminate the voltage unbalance at a certain node of the AC microgrid where a sensitive load is connected. The control parameters have been designed to guarantee stable operation and some dynamic specifications. In particular, the parameter $k_{w i}$ can be designed to provide a different contribution of the negativesequence inverter currents to reduce the inverters' negativesequence voltages. Selected experimental results demonstrate the enhancement of the power quality of the $\mathrm{AC}$ microgrid in different scenarios.

This work motivates several ideas for future research. First, the current distribution algorithm can be further exploited to not exceed the inverters' maximum current by introducing active-power injection curtailment, accepting a certain voltage unbalance in the sensitive load, or a combination of both approaches. Second, the possibility of eliminating the voltage unbalance in more than one sensitive node. Third, the extension of the control scheme to grid-forming inverters working in islanded-mode and powering unevenly distributed large single-phase loads, as a source of voltage unbalance. Finally, the study of voltage drops during grid faults in different cases (balanced-unbalanced sags, compliance with grid codes, online current limitation).

\section{REFERENCES}

[1] S. Chattopadhyay, M. Mitra, and S. Sengupta, "Electric power quality, " New York, NY, USA: Springer, 2011, pp. 1-182.

[2] A. von Jouanne and B. Banerjee, "Assessment of voltage unbalance," in IEEE Trans. Power Del., vol. 16, no. 4, pp. 782-790, Oct. 2001.

[3] D. Schwanz, F. Möller, S. K. Rönnberg, J. Meyer and M. H. J. Bollen, "Stochastic assessment of voltage unbalance due to single-phaseconnected solar power," IEEE Trans. Power Del, vol. 32, no. 2, pp. 852861, April 2017

[4] S. E. M. de Oliveira and J. O. R. P. Guimaraes, "Effects of voltage supply unbalance on AC harmonic current components produced by AC/DC converters," IEEE Trans. Power Del., vol. 22, no. 4, pp. 24982507, Oct. 2007.

[5] K. Lee, G. Venkataramanan and T. M. Jahns, "Modeling effects of voltage unbalances in industrial distribution systems with adjustablespeed drives," IEEE Trans. Ind. Appl, vol. 44, no. 5, pp. 1322-1332, Sept.-Oct. 2008

[6] M. Campbell, G. Arce, "Effect of motor voltage unbalance on motor vibration: Test and evaluation," IEEE Trans. Ind. Appl., vol. 54, no. 1, pp. $905-911$, Jan./Feb. 2018

[7] X. Du and R. Wang, "Control strategy of negative sequence current for the grid-connection converter under the unbalanced grid condition," 2015 34th Chinese Control Conference (CCC), Hangzhou, 2015, pp 9066-9071.

[8] G. F. Gontijo et al., "Modeling, control and experimental verification of a DFIG with a series-grid-side converter with voltage sag, unbalance and distortion compensation capabilities," IEEE Trans. Ind. Appl., early access.

[9] P. Pillay and M. Manyage, "California electricity situation," IEEE Power Eng. Review, vol. 21, no. 5, pp. 10-12, May 2001.
[10] EN B. 50160: Voltage characteristics of electricity supplied by public distribution systems. British Standards Institution; 2000.

[11] J. M. Guerrero, P. C. Loh, T. Lee, and M. Chandorkar, "Advanced control architectures for intelligent microgrids-Part II: Power quality, energy storage, and AC/DC microgrids," IEEE Trans. Ind. Electron., vol. 60, no. 4, pp. 1263-1270, April 2013.

[12] M. Castilla, J. Miret, A. Camacho, J. Matas and L. García de Vicuña," Voltage support control strategies for static synchronous compensators under unbalanced voltage sags," IEEE Trans. Ind. Electron. vol. 61, no. 2, pp. 808-820, Feb. 2014.

[13] A. M. Gee, F. Robinson, and W. Yuan, "A superconducting magnetic energy storage-emulator/battery supported dynamic voltage restorer," IEEE Trans. Energy Convers., vol. 32, no. 1, pp. 55-64, March 2017.

[14] Y. Naderi, S. H. Hosseini, S. G. Zadeh, B. Mohammadi-Ivatloo, J. C. Vasquez, and J. M. Guerrero, "An overview of power quality enhancement techniques applied to distributed generation in electrical distribution networks," Renew. Sustain. Energy Rev., vol. 93, pp. 201214, Oct. 2018

[15] X. Wang, J. M. Guerrero, F. Blaabjerg, and Z. Chen, "A review of power electronics based microgrids," J. Power Electron., vol. 12, no. 1, pp. 181- 192, Jan. 2012.

[16] J. Rocabert, A. Luna, F. Blaabjerg and P. Rodríguez, "Control of power converters in AC microgrids," IEEE Trans. Power Electron., vol. 27, no, 11, pp. 4734-4749, Nov. 2012.

[17] P.T. Cheng; C. Chen; T. L. Lee, and S.Y. Kuo, "A cooperative imbalance compensation method for distributed-generation interface converters," IEEE Trans. Ind. Appl., vol. 45, no. 2, pp. 805-815, MarApr 2009.

[18] M. Savaghebi, A. Jalilian, J. C. Vasquez, and J. M. Guerrero, "Autonomous voltage unbalance compensation in an islanded droopcontrolled microgrid," IEEE Trans. Ind. Electron., vol. 60, no. 4, pp. 1390-1402, Apr. 2013.

[19] F. Nejabatkhah, Y. W. Li and B. Wu, "Control strategies of three-phase distributed generation inverters for grid unbalanced voltage compensation," IEEE Trans. Power Electron., vol. 31, no. 7, pp. 52285241, July 2016.

[20] M. Moradi Ghahderijani, A. Camacho, C. Moreira, M. Castilla and L. G. De Vicuna, "Imbalance-voltage mitigation in an inverter-based distributed generation system using a minimum current-based control strategy," IEEE Trans. Power Del., early access.

[21] M. Velasco, P. Martí, A. Camacho, J. M. Rey, J. Miret and M. Castilla, "Enabling grid-feeding converters with a dissonant-resonant controller for negative-sequence voltage elimination," IEEE Trans. Power Electron., vol. 35, no. 4, pp. 4342-4352, April 2020.

[22] R. Teodorescu, F. Blaabjerg, M. Liserre and P. C. Loh, "Proportionalresonant controllers and filters for grid-connected voltage-source converters," IEEE Proceedings - Electric Power Applications, vol. 153, no. 5, pp. 750-762, September 2006.

[23] L. M. Wedepohl and L. Jackson, "Modified nodal analysis: an essential addition to electrical circuit theory and analysis," Engineering Science and Education Journal, vol. 11, no. 3, pp. 84-92, June 2002.

[24] IEEE Standard for Synchrophasor Measurements for Power Systems, IEEE Std C37.118.1-2011 (Revision of IEEE Std C37.118-2005), vol., no., pp.1-61, 28 Dec. 2011

[25] I. Serban, S. Céspedes, C. Marinescu, C. A. Azurdia-Meza, J. S. Gómez, and D. S. Hueichapan, "Communication requirements in microgrids: A practical survey," IEEE Access, vol. 8, pp. 47694-47712, Mar. 2020.

[26] Q. Zhou, M. Shahidehpour, A. Paaso, S. Bahramirad, A. Alabdulwahab and A. Abusorrah, "Distributed control and communication strategies in networked microgrids," IEEE Communications Surveys \& Tutorials Year: 2020 | Early Access Article Publisher: IEEE.

[27] W. P. M. H. Heemels, A. R. Teel, N. van de Wouw and D. Nešić, "Networked control systems with communication constraints: Tradeoffs between transmission intervals, delays and performance," IEEE Trans. Aut. Cont., vol. 55, no. 8, pp. 1781-1796, Aug. 2010.

[28] F. Blaabjerg, R. Teodorescu, M. Liserre and A. V. Timbus, "Overview of control and grid synchronization for distributed power generation systems," IEEE Trans. Ind. Electron, vol. 53, no. 5, pp. 1398-1409, Oct. 2006.

[29] P. Rodríguez, A. Luna, R. S. Muñoz-Aguilar, I. Etxeberria-Otadui, R. Teodorescu and F. Blaabjerg, "A stationary reference frame grid synchronization system for three-phase grid-connected power converters under adverse grid conditions," IEEE Trans. Power Electron, vol. 27, no. 1, pp. 99-112, Jan. 2012. 
[30] L. Harnefors, "Modeling of three-phase dynamic systems using complex transfer functions and transfer matrices," IEEE Trans. Ind. Electron., vol. 54, no. 4, pp. 2239-2248, Aug. 2007.

[31] S. Gataric and N. R. Garrigan, "Modeling and design of three-phase systems using complex transfer functions", $30^{\text {th }}$ Annual. IEEE Power Electron. Specialist Conf., vol. 2, 1999, pp. 691-697.

[32] M. Bodson and O. Kiselychnyk, "The complex Hurwitz test for the analysis of spontaneous self-excitation in induction generators," IEEE Trans. on Autom. Control, vol. 58, no. 2, pp. 449-454, Feb. 2013.

[33] A. Dòria-Cerezo and M. Bodson, "Design of controllers for electrical power systems using a complex root locus method," IEEE Trans. Ind. Electron., vol. 63, no. 6, pp. 3706-3716, June 2016.

[34] A. G. Yepes, et al., "Analysis and design of resonant current controllers for voltage-source converters by means of Nyquist diagrams and sensitivity function", IEEE Trans. Ind. Electron, vol. 58, No. 11, pp. 5231-5250, Nov. 2011. 